\title{
Gel Permeation Chromatography of Comb-Shaped Branched Polymers
}

\author{
Tadaya Kato, ${ }^{*}$ Akira Itsubo, ${ }^{* *}$ Yoshinori Yamamoto, \\ Teruo Fujimoto, and Mitsuru Nagasawa \\ Department of Synthetic Chemistry, Nagoya University, \\ Chikusa-ku, Nagoya, Japan.
}

(Received August 23, 1974)

\begin{abstract}
Comb-shaped branched polystyrenes prepared by anionic polymerization are fractionated carefully. The molecular weight, degree of branching and radius of gyration of those fractions are determined by light scattering. Gel permeation chromatogram (GPC) of those fractions are observed on toluene. If the radius of gyration of comb-shaped polystyrenes is plotted against retention volume, the data fit the same line as monodisperse linear polystyrenes. If the GPC data are replotted in the form of $\log [\eta] M v s$. retention volume, clear disagreement is found between both data, though the disagreement is not so large. A discussion is given to the reason for the disagreement.
\end{abstract}

KEY WORDS Gel Permeation Chromatogram / Comb-Shaped Polystyrenes / Calibration Curve / Hydrodynamic Volume / Radius of Gyration /

It was experimentally shown ${ }^{1-7}$ that the gel permeation chromatography (GPC) of both linear and branched polymers is governed by the product of intrinsic viscosity and molecular weight, $[\eta] M$. That is, the plot of $\log [\eta] M v s$. retention volume $V_{\mathrm{R}}$ can be used as a universal calibration curve, which is independent of polymer species and branching. This fact appears to imply that the mechanism of separation in GPC is also due to the hydrodynamic volume, though in the theory of Casassa and Tagami, ${ }^{8}$ the idea of hydrodynamic volume is not necessarily required to explain the mechanism of GPC. This paper is to present some GPC data of branched polymers which have more branches than the samples used in literature. ${ }^{1}$ To clarify the mechanism of separation in GPC, it may still be important to accumulate the GPC data of branched polymers for which both the radius of gyration and hydrodynamic radius are determined.

\footnotetext{
* Present address: Department of Industrial Chemistry, Mie University, Tsu-shi, Japan.

** Present address: Mitsubishi Yuka Co., Yokkaichi, Mie, Japan.
}

\section{EXPERIMENTAL}

\section{Samples}

The samples of comb-shaped branched polystyrenes used are the same as used in previous works. $^{9,10}$ That is, they were prepared by coupling chloromethylated monodisperse polystyrenes with monodisperse polystyryl anions. The products were fractionated stepwise in toluene-methanol system. And then each fraction was repeatedly refractionated from toluenemethanol or benzene-THF-methanol system to sharpen its molecular weight distribution, as explained previously. ${ }^{9}$ The preparation, purification, fractionation, and characterization of the samples were reported already.,10

The monodisperse linear polystyrenes used to determine the calibration curve were purchased from Pressure Chem. Co. Poly $(\alpha$-methylstyrenes) used in the molecular weight range higher than $10^{6}$ were prepared in this laboratory. ${ }^{11,12}$

\section{Solvents}

The solvents used for characterization of the samples were purified and fractionally distilled on $\mathrm{Na}$ metal wire just before use.

\section{Light Scattering Measurements \\ Measurements of light scattering were carried}


out by a modified Shimadzu light scattering photometer with unpolarized light of $436 \mathrm{~nm}$. The range of scattering angle used was from $18^{\circ}$ to $140^{\circ} .{ }^{12}$ The sample solution was clarified by filtration through $0.45-\mu \mathrm{m}$ Millipore filters. The relative intensity of scattered light is given by

$$
K c / R_{\theta}=1 / M_{w} P(\theta)+2 A_{2} c+\cdots
$$

where $K$ is an optical constant, $R_{\theta}$ is the Rayleigh ratio, $c$ is the polymer concentration $(\mathrm{g} / \mathrm{m} l), \quad M_{w}$ is the weight-average molecular weight, $A_{2}$ is the second virial coefficient and $P(\theta)$ is the particle scattering factor from which we can obtain the $z$-average statistical radius of polymer $\left\langle S^{2}\right\rangle_{z}{ }^{1 / 2}$.

The $P(\theta)$ function for polymers with the Gaussian distribution of segments around the center of gravity was calculated by Debye, ${ }^{13}$ as given by eq 2 , and that for comb-shaped polymers at the unperturbed state was calculated by Casassa and Berry, ${ }^{14}$ as given by eq 3 .

$$
\begin{aligned}
& P(\theta)=\left(2 / x^{2}\right)[\exp (-x)-1+x] \\
P(\theta)= & \left(\frac{2}{x^{2}}\right)\left[x-\left(1-\mathrm{e}^{-\lambda x}\right)+\left(1-\mathrm{e}^{-(1-\lambda) x / f}\right)\right. \\
& +\left\{f-\frac{2\left(1-\mathrm{e}^{-\lambda x}\right)}{\lambda x / f}\right\}+\left(1-\mathrm{e}^{-x(1-\lambda) / f}\right) \\
& \left.\times \frac{x-\left(1-\mathrm{e}^{-\lambda x}\right)}{\lambda^{2} x^{2} / f(f-1)}\right]
\end{aligned}
$$

where $x=16 \pi^{2}\left(n / \lambda_{0}\right)^{2}\left\langle S^{2}\right\rangle_{z} \sin ^{2}(\theta / 2)$ and $n$ is the refractive index of solvent and $\lambda_{0}$ is the wave length of light. The former is applicable to comb-shaped polymers if the segment distribution is of a Gaussian type. If $x$ is small, both equations can be expanded into

$$
P^{-1}(\theta)=1+\frac{1}{3} x+O\left(x^{2}\right)+\cdots
$$

However, the linear plot of $P^{-1}(\theta) v s . \sin ^{2}(\theta / 2)$ can be found only in poor solvents. In good solvents, the range of linear relationship is so limited that it is difficult to obtain $\left\langle S^{2}\right\rangle_{z}$ from the slope of $P^{-1}(\theta) v s \cdot \sin ^{2}(\theta / 2)$ plot. Our discussion on the determination of radius of gyration of linear polymers in good solvents was given in a previous paper. ${ }^{11}$ In this work, $\left\langle S^{2}\right\rangle_{z}$ of comb-shaped polymers in good solvent is determined so that the best agreement may be found between eq 3 and experimental values in a range of low $\theta$.

Examples of $P^{-1}(\theta) v s \cdot \sin ^{2}(\theta / 2)$ plot for combshaped polystyrenes in poor solvents were shown in a previous paper. ${ }^{9}$ Figure 1 shows an example of the plot in toluene at $25^{\circ} \mathrm{C}$. The solid line shows the theoretical curve of Casassa and Berry which shows the best agreement with experimental data. The broken line shows the theoretical curve of Debye with $\left\langle S^{2}\right\rangle_{z}$ chosen to give good agreement between experimental data and eq 2. Both theories give almost the same radius of gyration; $8.8 \times 10^{-6} \mathrm{~cm}$ from eq 2 and $8.5 \times 10^{-6} \mathrm{~cm}$ from eq 3 . In the range of high $\sin ^{2}(\theta / 2)$, both theoretical curve shows deviation from experimental data. The deviations may be caused by either the non-Gaussian distribution of segments and/or polydispersity.

\section{Viscosity Measurements}

The limiting viscosity numbers of branched

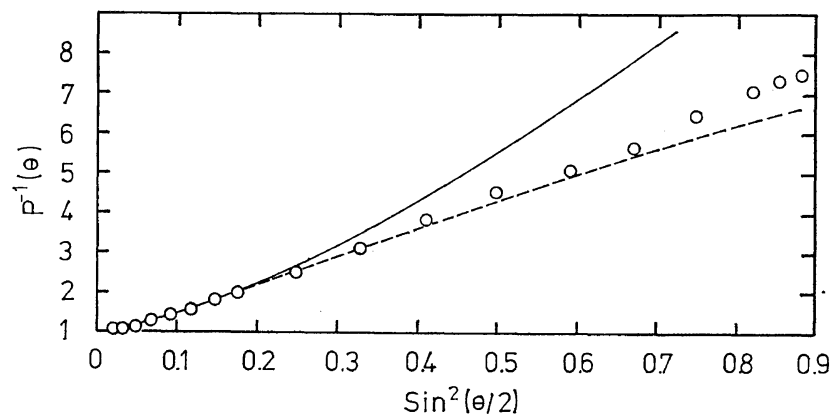

Figure 1. Determination of radius of gyration by light scattering: Sample, L-2; solvent, toluene; temp, $25^{\circ} \mathrm{C}$. The solid line shows the calculated values of Casassa and Berry with $\left\langle S^{2}\right\rangle=7.24 \times 10^{-11}$. The broken line shows those of Debye with $\left\langle S^{2}\right\rangle=7.76 \times 10^{-11}$. 
Gel Permeation Chromatography of Comb-Shaped Branched Polymers

polymers were determined in a Ubbelohde capillary viscometer, for which the kinetic energy correction was negligible. All experimental procedures are the same as in a previous paper. ${ }^{9}$

\section{GPC Measurements}

A high speed liquid chromatograph HLC-801A, manufactured by Toyo Soda MFG. Co., Ltd. was operated at $40^{\circ} \mathrm{C}$ on toluene with a solvent flow rate of $1.0 \mathrm{ml} / \mathrm{min}$, equipped three TSK G6000H8 columns $\left(10^{6} \AA ; 2 \mathrm{ft} \times 3 / 8\right.$ inch column outside diam.). The concentration of the sample injected was $0.5 \mathrm{mg} / \mathrm{m} l$ and the by-pass injector roop is $0.282 \mathrm{ml}$. The column packing material was porous cross-linked polystyrene gel which was synthesized from styrene monomer and divinylbenzene mixture by Toyo Soda Co. The theoretical plate numbers of each column reported by the manufacturer were more than 4000 plates/ft.

The retention volume $V_{\mathrm{R}}$, which is the liquid volume flowing through the column from the sample injection to the peak maximum, is expressed by counting of liquid drop. One count corresponds to $1.0 \mathrm{~m} l$. As the molecular weight distribution of each fraction is fairly sharp and also symmetrical, it may be assumed that the effect of peak broadening on the position of peak maximum is negligible, i.e., the peak maximum corresponds to $M_{w} \cdot{ }^{15}$

\section{RESULTS}

The molecular characteristics of the combshaped and linear samples used are shown in Table I and II, respectively. Some values in the tables were already reported in previous paper. $^{9,11}$ Figure 2 shows the double logarithmic plot of $\left\langle S^{2}\right\rangle_{z} v s . M_{w}$. The solid straight lines give the data for linear polystyrenes in toluene $25^{\circ} \mathrm{C}$. The radii of gyration of comb-shaped polystyrenes are much smaller than those of linear polystyrenes with the same molecular weights as the comb-shaped polystyrenes.

Table I. Molecular characteristics of comb-shaped polystyrene samples

\begin{tabular}{|c|c|c|c|c|c|c|c|}
\hline Samples & $\begin{array}{c}\text { Mol wt of } \\
\text { parent } \\
\text { polymers, } \\
M_{0} / 10^{4}\end{array}$ & $\begin{array}{l}\text { Mol wt } \\
\text { of a } \\
\text { branch, } \\
M_{\mathrm{b}} / 10^{4}\end{array}$ & $\begin{array}{l}\text { Number of } \\
\text { branches }\end{array}$ & $M_{w} / 10^{6}$ & $\begin{array}{l}\left\langle S^{2}\right\rangle_{z} / 10^{-11} \\
\text { in toluene }\end{array}$ & $\begin{array}{c}{[\eta]} \\
\text { in toluene, } \\
25^{\circ} \mathrm{C}\end{array}$ & $\begin{array}{c}V_{R}, \\
\text { count }\end{array}$ \\
\hline $\mathrm{F}-11$ & $9.5_{8}$ & $6.5_{1}$ & 10.1 & $0.7_{6}$ & $0.7_{1}$ & $0.65_{8}$ & $40.9_{0}$ \\
\hline $\mathrm{H}-4$ & \multirow{3}{*}{$9.7_{6}$} & \multirow{3}{*}{12.9} & 26.6 & $3.5_{3}$ & $2.5_{0}$ & $1.6_{2}$ & $36.0_{3}$ \\
\hline 5 & & & 22.6 & $3.0_{1}$ & $2.3_{6}$ & $1.4_{3}$ & $36.5_{6}$ \\
\hline 6 & & & 18.8 & $2.4_{3}$ & $1.8_{2}$ & $1.3_{3}$ & 36.89 \\
\hline $\mathrm{I}-4$ & \multirow{3}{*}{$9.7_{6}$} & \multirow{3}{*}{17.6} & 28.7 & $4.1_{5}$ & $3.5_{4}$ & $1.9_{0}$ & $35.5_{0}$ \\
\hline 7 & & & 17.8 & $3.2_{4}$ & $2.7_{3}$ & $1.6_{8}$ & $36.3_{8}$ \\
\hline 9 & & & 9.9 & $1.8_{4}$ & $1.7_{4}$ & $1.4_{5}$ & - \\
\hline $\mathrm{J}-2$ & \multirow{3}{*}{$9.7_{6}$} & \multirow{3}{*}{35.8} & 9.3 & $3.4_{1}$ & $5.9_{1}$ & $2.6_{6}$ & $35.2_{4}$ \\
\hline 5 & & & 4.6 & $1.7_{5}$ & $3.2_{8}$ & $2.3_{2}$ & $36.5_{8}$ \\
\hline 8 & & & 2.7 & $0.9_{6}$ & - & $1.9_{1}$ & $38.5_{3}$ \\
\hline $\mathrm{K}-1$ & \multirow{5}{*}{$8.8_{3}$} & \multirow{5}{*}{14.7} & 103 & 16.1 & - & $3.8_{6}$ & $32.9_{4}$ \\
\hline 2 & & & 88 & 13.9 & - & $3.7_{5}$ & $33.6_{0}$ \\
\hline 3 & & & 56 & $9.1_{8}$ & - & $3.5_{0}$ & $33.6_{7}$ \\
\hline 4 & & & 42 & $7.0_{6}$ & - & $3.0_{z}$ & $33.7_{9}$ \\
\hline 5 & & & 33 & 5.88 & - & $2.9_{9}$ & $34.0_{0}$ \\
\hline L-1 & \multirow{5}{*}{$3.6_{8}$} & \multirow{5}{*}{$6.3_{2}$} & 192 & 12.5 & 10.2 & $2.3_{7}$ & $33.8_{3}$ \\
\hline 2 & & & 149 & $9.8_{0}$ & $7.2_{4}$ & $2.1_{0}$ & 34.49 \\
\hline 3 & & & 132 & $8.6_{9}$ & 6.59 & $1.9_{4}$ & $35.3_{5}$ \\
\hline 4 & & & 117 & $7.7_{5}$ & $5.4_{3}$ & $1.8_{6}$ & $35.0_{0}$ \\
\hline 5 & & & 87 & $5.8_{8}$ & $4.1_{8}$ & $1.7_{3}$ & $35.5_{1}$ \\
\hline
\end{tabular}


Table II. Molecular characteristics of linear polymers

\begin{tabular}{|c|c|c|c|c|}
\hline Samples & $M_{w} / 10^{4}$ & $\left\langle S^{2}\right\rangle / 10^{-11}$ & $\begin{array}{c}{[\eta]} \\
\text { in toluene, } \\
25^{\circ} \mathrm{C}\end{array}$ & $\begin{array}{l}V_{\mathrm{R}}, \\
\text { count }\end{array}$ \\
\hline PS-530 & $53_{2}$ & 10.2 & $8.3_{8}$ & $32.4_{5}$ \\
\hline PS-180 & $19_{3}$ & $4.9_{1}$ & $3.2_{1}$ & $34.4_{6}$ \\
\hline PS-86 & 90.1 & $1.7_{9}$ & $2.0_{3}$ & $37.9_{0}$ \\
\hline PS-67 & $(67)^{a}$ & - & 1.70 & $38.9_{0}$ \\
\hline PS-41 & 40.5 & - & $1.25_{6}$ & $41.1_{8}$ \\
\hline PS-16 & $(16.0)$ & - & $0.65_{4}$ & $45.2_{0}$ \\
\hline $\mathrm{P} \alpha \mathrm{MS}-14$ & $74_{7}$ & 18.7 & 9.4 & $31.8_{2}$ \\
\hline $\mathrm{P} \alpha \mathrm{MS}-13$ & 330 & $7.3_{3}$ & $4.6_{5}$ & $33.9_{2}$ \\
\hline $\mathrm{P} \alpha \mathrm{MS}-12$ & $18_{2}$ & $3.9_{7}$ & $3.17_{5}$ & $35.7_{7}$ \\
\hline P $\alpha$ MS-9 & $11_{9}$ & $2.3_{0}$ & $2.42_{4}$ & $36.7_{3}$ \\
\hline $\mathrm{P} \alpha \mathrm{MS}-7$ & 69.4 & $1.1_{9}$ & 1.49 & $38.8_{1}$ \\
\hline P $\alpha$ MS- 6 & 44.4 & $0.73_{1}$ & $1.04_{2}$ & $41.6_{8}$ \\
\hline P $\alpha$ MS-5 & 34.2 & $0.49_{8}$ & $0.89_{5}$ & $42.6_{1}$ \\
\hline
\end{tabular}

a ( ) are the values reported by the manufacturer.

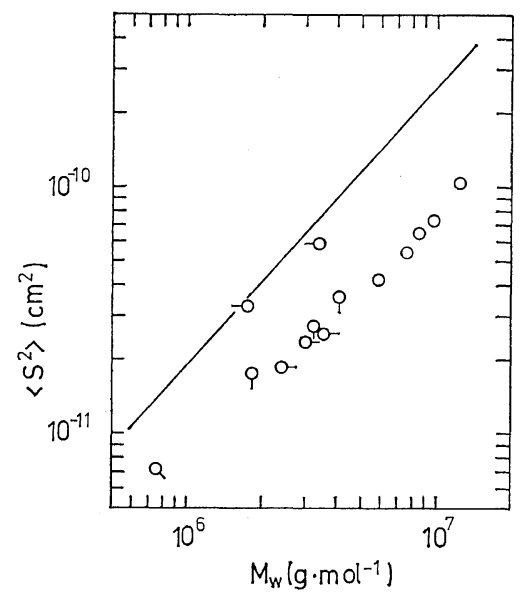

Figure 2. Molecular weight dependence of radius of gyration of comb-shaped polystyrenes. Solid lines show the radius of gyration of monodisperse polystyrenes in toluene $25^{\circ} \mathrm{C} .11$

Sample Nos. J-2 and J-5, which show behavior different from other branched polymers, have much smaller number of branches than the others. Figure 3 shows the relationship between molecular weight and retention volume for both linear and branched polymers. The retention time for a comb-shaped polystyrene is much longer than that for the corresponding linear polystyrene. $^{1}$

The data of $[\eta] M$ for comb-shaped polymers

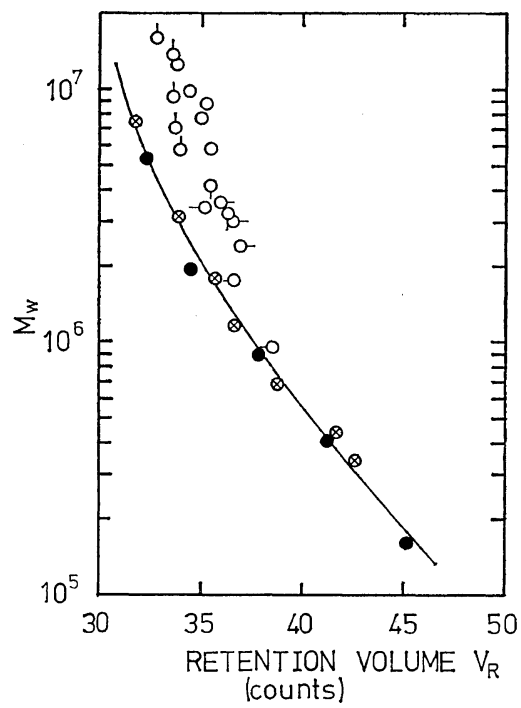

Figure 3. Relationship between GPC peak retention volume and molecular weight. Open and filled circles show the data for comb-shaped and monodisperse linear polystyrenes, respectively: $Q$, samples F; $0-\mathbf{H}$; $ᄋ, \mathbf{I} ;-0, \mathbf{J} ; \quad+, \mathbf{K} ; 0, \mathbf{L} . \otimes$ denotes the data of monodisperse linear poly- $\alpha-$ methylstyrenes.

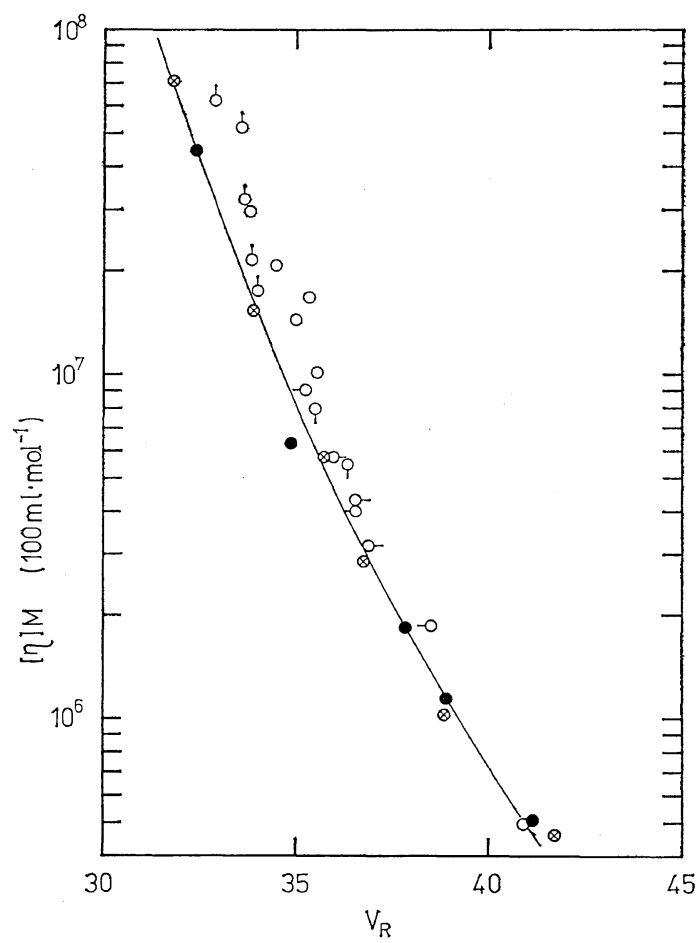

Figure 4. Comparison of the data for comb-shaped polystyrenes with the universal curve of GPC. Notations are the same as in Figure 3. 
Gel Permeation Chromatography of Comb-Shaped Branched Polymers

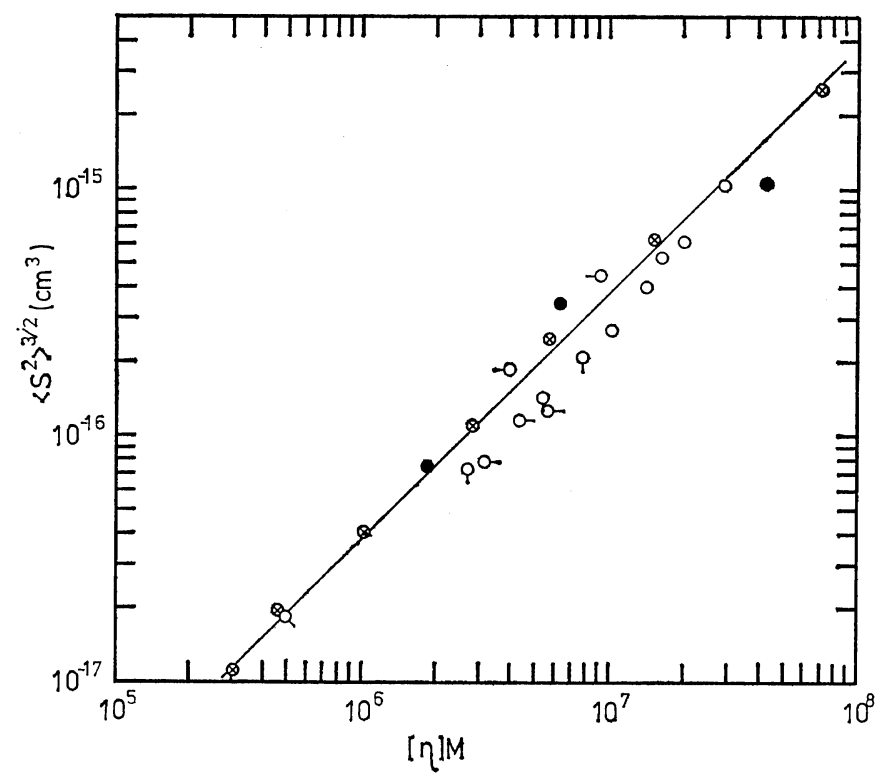

Figure 5. Relationship between radius of gyration and hydrodynamic volume.

Notations are the same as in Figure 3.

are plotted against retention volume $V_{\mathrm{R}}$ in comparison with the universal curve for linear polymers in Figure 4. The calibration curves for monodisperse polystyrene and $\operatorname{poly}(\alpha-$ methylstyrenes) agree very well. However, the data for comb-shaped polymers slightly but clearly deviate from the line for linear polymers.

If the comb-shaped polymers behave as hydrodynamically nondraining spheres in solution as linear polymers do, the following wellknown relationship of Flory and Fox ${ }^{16}$ should hold for comb-shaped polymers, too.

$$
[\eta]=\Phi \frac{\left\langle S^{2}\right\rangle^{3 / 2}}{M}
$$

The constant $\Phi$ for comb-shaped polymers may not be equal to that for linear polymers, but would not differ much. This speculation was experimentally proved at least in $\Theta$ solvents. $^{9}$ If $\Phi$ is the same for both comb-shaped and linear polymers in good solvents, too, both hydrodynamic volumes, $[\eta] M$, for linear and comb-shaped polymers should fit the same line. Figure 5 shows the plot of $\log \left\langle S^{2}\right\rangle_{z}{ }^{3 / 2} v s$. $\log [\eta] M$ for both comb-shaped and linear polymers. The data for comb-shaped polymers are slightly below the line for linear polymers.

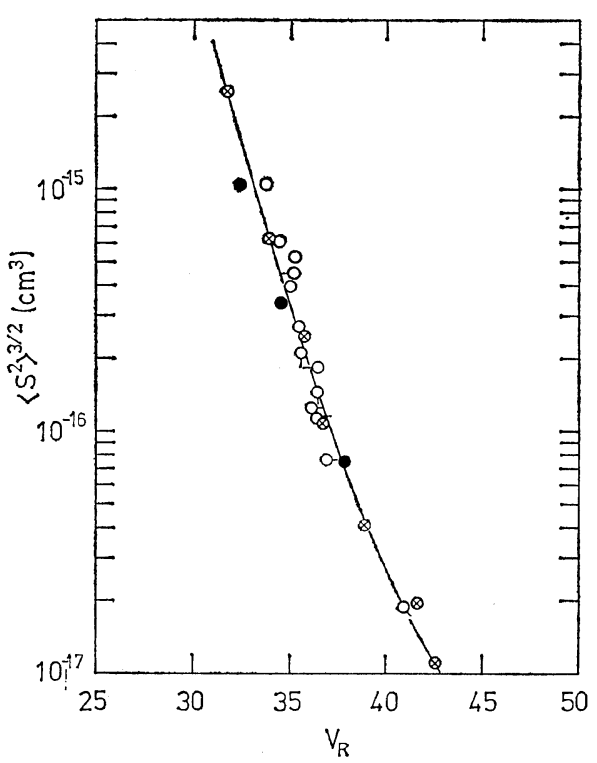

Figure 6. Relationship between radius of gyration and GPC retention volume. Notations are the same as in Figure 3.

Figure 6 shows the relationship between $\log \left\langle S^{2}\right\rangle_{z}{ }^{3 / 2} v s . V_{\mathrm{R}}$ for linear and comb-shaped polymers. The experimental data for combshaped polymers agree with the data for linear 
polymers. Although the deviation in Figures 4 and 5 are so small that it is difficult to have a clear conclusion without the slightest ambiguity, it is our opinion that if we use the radius of gyration $\left\langle S^{2}\right\rangle_{z}{ }^{1 / 2}$, the GPC data of comb-shaped polymers fit the universal curve (Figure 6), whereas if we employ the hydrodynamic volumes $[\eta] M$, they may show deviation from the universal curve (Figure 4).

\section{DISCUSSION}

The degree of branching is usually expressed by a parameter $g_{S}$ which is defined by the ratio of the unperturbed radii of gyration of linear and branching polymers having the same molecular weight. That is

$$
\left\langle S^{2}\right\rangle_{0 \mathrm{~b}} /\left\langle S^{2}\right\rangle_{0 l}=g_{S}
$$

where $\mathrm{b}$ and $l$ denote the branched and linear polymers and $\left\langle S^{2}\right\rangle_{0}$ denotes the square radius of gyration at the unperturbed state. If we know the type of branching such as star-shaped, combshaped or randomly branched polymers, $g_{S}$ can be expressed in terms of molecular characteristics such as degree of branching, molecular weight of branch, etc.

A similar parameter $g_{\eta}$ can be defined between the hydrodynamic volumes, i.e., limiting viscosity numbers of linear and branched polymers:

$$
[\eta]_{0 \mathrm{~b}} /[\eta]_{0 l}=g_{\eta}
$$

where $[\eta]_{0}$ denotes the limiting viscosity number at the $\Theta$ state. If the hydrodynamic radius is proportional to the radius of gyration in both linear and branched polymers, there must be the following relationship between $g_{S}$ and $g_{\eta}$.

$$
g_{\eta}=g_{S}{ }^{3 / 2}
$$

For star-shaped and randomly branched polymers, however, Zimm, Kilb, ${ }^{17}$ Kurata and Fukatsu ${ }^{18}$ gave the following relationship

$$
g_{\eta}=g_{S}^{1 / 2} \text {. }
$$

Our previous work shows that eq 8 holds for our comb-shaped polymers at the $\Theta$ state, despite that they are almost star-like in shape. ${ }^{9}$ The present experimental results also appear to support this conclusion. Figures 4 and 5 imply that the hydrodynamic radius is approximately proportional to radius of gyration in a good solvent.

Various reasons may be conceivable for small deviation found in Figure 4.

(1) GPC is carried out at a finite polymer concentration, whereas $[\eta]$ is the value at the infinite dilution. If the polymer concentration dependence of the hydrodynamic volume of a polymer is different from that of ordinary polymers, the data for the polymers may show deviation from the universal curve. ${ }^{19}$ However, the effect of polymer concentration dependence may not be the reason for the discrepancy in Figure 4. The concentration $0.5 \mathrm{mg} / \mathrm{ml}$ used here is believed to be low enough to neglect this effect. ${ }^{2021}$ Moreover, the deviation by this effect should appear in both Figures 4 and 6 .

(2) The universal curve is obtained when the polymers used are all monodisperse or when the effect of polyderpersity is equal for all samples. ${ }^{22}$ In general, [ $\eta]$ and $\left\langle S^{2}\right\rangle_{z}$ of polydisperse samples are larger than those of monodisperse samples having the same weight-average molecular weights. ${ }^{23}$ Moreover, the effect of polydispersity on $\left\langle S^{2}\right\rangle_{z}$ is more remarkable than that on [ $\left.\eta\right]$. Therefore, if a sample has a broad molecular weight distribution, and also if GPC is determined by radius of gyration, the data for the polydisperse sample may show deviation from the universal curve. However, this cannot be the reason for the deviation in Figure 4, since no deviation is found in Figure 6. Moreover, the present samples have fairly sharp molecular weight distributions though they are fractionated samples. ${ }^{9}$

(3) GPC is generally carried out in good solvents. Therefore, the polymer coils are expanded due to excluded volume effect. If the expansion factor of a polymer behaves differently from those of ordinary polymers, the Flory constant $\Phi$ may become different from the ordinary value $\left(3.1 \times 10^{22}\right)$ in good solvents. ${ }^{3,24}$ This excluded volume effect may be important in the GPC of comb-shaped polymers. Since the present samples have many branches, the expansion of the branched polymers due to excluded volume effect may be quite different from that of linear polymers as was reported by Candau, Rempp, and Benoit. ${ }^{25}$ That is, the Flory constant $\Phi$ for branched polymers in good 
solvents may be different from the ordinary value. It is likely that the deviations observed in both Figures 4 and 5 are due to the difference in the excluded volume effect of linear and branched polymers. Entirely the same prediction was already given by Pannell. ${ }^{26}$ Our discussion on the expansion factor of branched polymers will be given in a separate paper.

However, the deviation found in Figures 4 and 5 is not so large despite that the present samples have high densities of branches. It is understandable that other branched polymers with low density of branches do not show deviation from the universal curve., ${ }^{1,6,7}$

Acknowledgement. We wish to thank Dr. I. Noda for his helpful discussion and Mr. N. Kato for his help in GPC measurements.

\section{REFERENCES}

1. Z. Grubisic, P. Rempp, and H. Benoit, Polymer Letters, 5, 753 (1967); H. Benoit, Z. Grubisic, P. Rempp, D. Decker, and J. G. Zilloix, J. Chem. Phys., 63, 1507 (1966).

2. L. Wild and R. Guliana, J. Polym. Sci., Part A-2, 5, 1087 (1967).

3. H. Coll and D. K. Gilding, ibid., Part A-2, 8, 89 (1970).

4. M. Kurata, M. Abe, M. Iwama, and M. Matsushima, Polymer J., 3, 729 (1972); M. Kurata, H. Okamoto, M. Iwama, M. Abe, and T. Honma, ibid., 3, 740 (1972).

5. K. Kamada and H. Sato, ibid., 2, 489 (1971); ibid., 2, 593 (1971); K. Kamada, T. Masuzawa, and T. Yoshiwara, Kobunshi Kagaku (Chem. High Polymers), 29, 566 (1972).

6. T. Masuda, Y. Ohta, and S. Onogi, Macromolecules, 4, 763 (1971).

7. J. Herz, M. Hert, and C. Strazielle, Macromol. Chem., 160, 213 (1972).
8. E. F. Casassa and Y. Tagami, Macromolecules, 2, 14 (1969); E. F. Casassa, J. Phys. Chem., 75, 3929 (1971).

9. I. Noda, T. Horikawa, T. Kato, T. Fujimoto, and M. Nagasawa, Macromolecules, 3, 795 (1970).

10. T. Fujimoto, H. Narukawa, and M. Nagasawa, ibid., 3, 57 (1970).

11. T. Kato, K. Miyaso, I. Noda, T. Fujimoto, and M. Nagasawa, Macromolecules, 3, 777 (1970).

12. T. Kato, K. Miyaso, and M. Nagasawa, $J$. Phys. Chem., 72, 2161 (1968).

13. P. Debye, J. Phys. Colloid Chem., 51, 18 (1947).

14. E. F. Casassa and G. C. Berry, J. Polym. Sci., Part A-2, 4, 881, (1966); E. F. Casassa and Y. Tagami, ibid., Part A-2, 6, 63 (1968).

15. H. L. Berger and A. R. Schultz, ibid., Part A-3, 3643 (1965).

16. P. J. Flory and T. G Fox, J. Amer. Chem. Soc., 73, 1904 (1951); P. J. Flory, "Principles of Polymer Chemistry," Cornell Univ. Press, Ithaca, N. Y., 1953.

17. C. D. Thurmond and B. H. Jimm, J. Polym. Sci., 8, 477 (1952); B. H. Zimm and R. W. Kilb, ibid., 37, 19 (1959).

18. M. Kurata and M. Fukatsu, J. Chem. Phys. 41, 2934 (1964).

19. A. Rudin and H. L. W. Hoegy, J. Polym. Sci., Part A-1, 10, 217 (1972).

20. K. P. Goets, R. S. Porter, and J. F. Johnson, ibid., Part A-2, 9, 2255 (1971).

21. Y. Kato and T. Hashimoto, Kobunshi Kagaku (Chem. High Polymers), 30, 107 (1973).

22. B. A. Whitehouse, Macromolecules, 4, 463 (1971).

23. E. P. Otocka, R. J. Roe, M. Y. Hellman, and P. M. Muglia, Macromolecules, 4, 507 (1971).

24. J. V. Dawkins and M. Hemming, Macromol. Chem., 155, 75 (1972).

25. F. Candau, P. Rempp, and H. Benoit, Macromolecules, 5, 627 (1972).

26. J. Pannel, Polymer, 13, 277 (1972). 